Article

\title{
New Characterizations of the Clifford Torus and the Great Sphere
}

\author{
Sun Mi Jung ${ }^{1}$, Young Ho Kim ${ }^{1, *}$ and Jinhua Qian ${ }^{2}$ \\ 1 Department of Mathematics, Kyungpook National University, Daegu 41566, Korea \\ 2 Department of Mathematics, Northeastern University, Shenyang 110004, China \\ * Correspondence: yhkim@knu.ac.kr; Tel.: +82-53-950-7325
}

Received: 2 August 2019; Accepted: 23 August 2019; Published: 27 August 2019

\begin{abstract}
In studying spherical submanifolds as submanifolds of a round sphere, it is more relevant to consider the spherical Gauss map rather than the Gauss map of those defined by the oriented Grassmannian manifold induced from their ambient Euclidean space. In that sense, we study ruled surfaces in a three-dimensional sphere with finite-type and pointwise 1-type spherical Gauss map. Concerning integrability and geometry, we set up new characterizations of the Clifford torus and the great sphere of 3-sphere and construct new examples of spherical ruled surfaces in a three-dimensional sphere.
\end{abstract}

Keywords: Clifford torus; spherical Gauss map; finite-type; pointwise 1-type spherical Gauss map

\section{Introduction}

In the 1960s, T. Takahashi proved that an isometric immersion $x: M \rightarrow \mathbb{E}^{m}$ of a Riemannian manifold $M$ into a Euclidean space $\mathbb{E}^{m}$ satisfies $\Delta x=\lambda x(\lambda \neq 0)$ if, and only if, it is part of a hypersphere or a minimal submanifold of a hypersphere, where $\Delta$ denotes the Laplacian of $M$ [1] . Generalizing such an eigenvalue problem of immersion, B.-Y. Chen introduced the notion of finite-type immersion of a Riemannian manifold $M$ into a Euclidean space $\mathbb{E}^{m}$ in the late 1970s. Since then, it has been used as a remarkably useful tool in differential geometry to classify and characterize many manifolds including minimal submanifolds in $\mathbb{E}^{m}$. In particular, minimal submanifolds of Euclidean space are considered as a spacial case of submanifolds of the finite-type, in fact they are of 1-type [2,3]. Thanks to Nash's embedding theorem of Riemannian manifolds, it has been a natural consideration of Riemannian manifolds as submanifolds in Euclidean space along with the notion of finite-type immersion.

A ruled surface or a ruled submanifold of Euclidean space or Minkowski space is one of the most natural geometric objects in classical differential geometry which has been examined under finite-type related geometric conditions [4-7]. The well-known Catalan's Theorem says that the only minimal ruled surfaces in Euclidean 3-space are the planes and the helicoids. A general ruled submanifold of a smooth manifold is defined by a foliation of totally geodesic submanifolds along a smooth curve. In [8], it was shown that a regular and connected ruled surface $M$ in $\mathbb{S}^{3}$ is of finite-type if and only if it is an open part of a ruled minimal surface in $\mathbb{S}^{3}$ or an open part of a Riemannian product of two circles of different radii.

Such a theory of finite-type immersion in a Riemannian sense was naturally extended to an isometric immersion of a manifold $M$ into a pseudo-Euclidean space $\mathbb{E}_{s}^{m}$ with index $s$ and the smooth functions defined on a submanifold in $\mathbb{E}^{m}$ or $\mathbb{E}_{s}^{m}$. In particular, the Gauss map on a submanifold in $\mathbb{E}^{m}$ or $\mathbb{E}_{s}^{m}$ is the most interesting and useful object which involves rich geometrical and topological properties on the submanifold. 
Regarding the Gauss map of finite-type, B.-Y. Chen and P. Piccinni initiated the study of submanifolds with a finite-type Gauss map in Euclidean space [9]. Many works about submanifolds in $\mathbb{E}^{m}$ or $\mathbb{L}^{m}$ with a finite-type Gauss map have been achieved [9-13]. In [10], C. Baikoussis showed that the only ruled submanifolds $M^{n+1}$ in Euclidean space $\mathbb{E}^{m}$ with a finite-type Gauss map are the cylinders over curves of finite-type and the $(n+1)$-dimensional Euclidean spaces. Ruled surfaces and ruled submanifolds with a finite-type Gauss map in Minkowski space were examined and completely classified in [6,14-17].

During the last ten years or so, the present authors et. al have worked on submanifolds of Euclidean or pseudo-Euclidean space which look similar to those of 1-type Gauss maps, which is called pointwise 1-type. For example, the Gauss maps $G$ of the helicoid and the right cone in $\mathbb{E}^{3}$ satisfy $\Delta G=f(G+\mathbf{C})$ for a nonzero smooth function $f$ and a constant vector $\mathbf{C}$ (cf. [18-20]). Since it was introduced in [18], many works concerning pointwise 1-type Gauss maps were established in [19-24]. In [22], the authors showed that the ruled submanifold $M$ in $\mathbb{E}^{m}$ is minimal if, and only if, the Gauss map $G$ of $M$ is pointwise 1-type of the first kind. The classification theorems of ruled submanifolds in the Euclidean space $\mathbb{E}^{m}$ and the Minkowski space $\mathbb{L}^{m}$ with pointwise 1-type Gauss maps were completed $[25,26]$.

On the other hand, one of the important manifolds in differential geometry is a sphere or a spherical submanifold. Regarding such manifolds, Obata studied the spherical Gauss map for a spherical submanifold $M$ in the unit hypersphere $\mathbb{S}^{m}\left(\subset \mathbb{E}^{m+1}\right)$ [27]. The set $S$ of all the great $n$-spheres in $\mathbb{S}^{m}$ is naturally identified with the oriented Grassmannian manifold of $(n+1)$-planes through the center of $\mathbb{S}^{m}$ in $\mathbb{E}^{m+1}$ because such $(n+1)$-planes determine unique great $n$-spheres and conversely [27]: A spherical Gauss map of an immersion $x$ of a Riemannian manifold $M$ into $\mathbb{S}^{m}$ is a map of $M$ into the oriented Grassmannian manifold $G(n+1, m+1)$ which assigns to each point $p$ of $M$ the great $n$-sphere tangent to $M$ at $x(p)$, or the $(n+1)$-plane spanned by the tangent space of $M$ at $x(p)$ and the normal to $\mathbb{S}^{m}$ at $x(p)$ in $\mathbb{E}^{m+1}$. Granted, the spherical Gauss map is more meaningful than the classical Gauss map in the study of spherical submanifolds (cf. $[28,29])$. Extending the notion of finite-type Gauss maps of submanifolds of Euclidean space in the usual sense, B.-Y. Chen and H.-S. Lue initiated the study of spherical submanifolds with finite-type spherical Gauss maps and obtained several fundamental results in this respect [28]. Recently, some works on spherical submanifolds with low-type spherical or pseudo-spherical Gauss maps have been made [30-32].

In this article, we study ruled surfaces in $\mathbb{S}^{3}$ by means of the spherical Gauss map to characterize the Clifford torus and the great sphere in the three-dimensional unit sphere $\mathbb{S}^{3}$.

In the present paper, all geometric objects are assumed to be smooth, and manifolds under consideration are connected unless otherwise stated.

\section{Preliminaries}

Let $x: M \rightarrow \mathbb{S}^{m-1}$ be an isometric immersion of an $n$-dimensional Riemannian manifold $M$ into a unit sphere $\mathbb{S}^{m-1}\left(\subset \mathbb{E}^{m}\right)$. We identify $x$ with its position in a vector field. Let $\left(y_{1}, y_{2}, \ldots, y_{m}\right)$ be a local coordinate system of $M$ in $\mathbb{S}^{m-1}$. For the components $g_{i j}$ of the Riemannian metric $\langle\cdot, \cdot\rangle$ on $M$ induced from that of $\mathbb{S}^{m-1}$, we denote by $\left(g^{i j}\right)$ (respectively, $\mathcal{G}$ ) the inverse matrix (respectively, the determinant) of the matrix $\left(g_{i j}\right)$. Then the Laplace operator $\Delta$ on $M$ is defined by

$$
\Delta=-\frac{1}{\sqrt{\mathcal{G}}} \sum_{i, j} \frac{\partial}{\partial y_{i}}\left(\sqrt{\mathcal{G}} g^{i j} \frac{\partial}{\partial y_{j}}\right) .
$$

An immersion $x$ of a manifold $M$ into $\mathbb{S}^{m-1}$ is said to be of finite-type if its position vector field $x$ can be expressed as a finite sum of spectral decomposition as follows

$$
x=x_{0}+x_{1}+\cdots+x_{k}
$$


for some positive integer $k$, where $x_{0}$ is a constant vector, and $\Delta x_{i}=\lambda_{i} x_{i}$ for some $\lambda_{i} \in \mathbb{R}, i=1, \ldots, k$. If $\lambda_{1}, \ldots, \lambda_{k}$ are mutually different, $M$ is said to be of $k$-type. Similarly, a smooth map $\phi$ on an $n$-dimensional submanifold $M$ of $\mathbb{S}^{m-1}$ is said to be of finite-type if $\phi$ is a finite sum of $\mathbb{E}^{m}$-valued eigenfunctions of $\Delta$. In particular, we say that a smooth map $\phi$ is harmonic if $\Delta \phi=0$. If the manifold $M$ is compact without boundary, a harmonic map is constant and thus it is of finite-type. In general, harmonic smooth map is not necessarily of finite-type if $M$ is not compact.

Let $\Pi$ be an oriented $n$-plane in $\mathbb{E}^{m}$ and $e_{1}, \ldots, e_{n}$ an orthonormal basis of $\Pi$. If we identify an oriented $n$-plane $\Pi$ with a decomposable $n$ vector $e_{1} \wedge \cdots \wedge e_{n}$ defined by the exterior algebra in a natural way, the oriented Grassmannian manifold $G(n, m)$ can be regarded as the set of all oriented $n$-planes in $\mathbb{E}^{N}=\Lambda^{n} \mathbb{E}^{m}$, where $N=\left(\begin{array}{c}m \\ n\end{array}\right)$. Moreover, we can define an inner product in $G(n, m)$ by

$$
\ll e_{i_{1}} \wedge \cdots \wedge e_{i_{n}}, e_{j_{1}} \wedge \cdots \wedge e_{j_{n}} \gg=\operatorname{det}\left(\left\langle e_{i_{l}}, e_{j_{k}}\right\rangle\right)
$$

for two vectors $e_{i_{1}} \wedge \cdots \wedge e_{i_{n}}$ and $e_{j_{1}} \wedge \cdots \wedge e_{j_{n}}$ in $\mathbb{E}^{N}$.

From now on we assume that the unit sphere $\mathbb{S}^{m-1}$ is centered at the origin in $\mathbb{E}^{m}$. We identify each tangent vector $X$ of $M$ in $\mathbb{S}^{m-1}$ with the differential $d x(X)$.

For a spherical submanifold $M$ in $\mathbb{S}^{m-1}$, the position vector $x$ of each point $p$ of $\mathbb{S}^{m-1}$ and an orthonormal basis $\left\{e_{1}, e_{2}, \ldots, e_{n}\right\}$ of the tangent space $T_{p} M$ determine an oriented $(n+1)$-plane in $\mathbb{E}^{m}$. Thus, we can have a map

$$
G: M \rightarrow G(n+1, m)
$$

via $G(p)=x \wedge e_{1} \wedge \cdots \wedge e_{n}$. We call $G$ the spherical Gauss map of $M$ in $\mathbb{S}^{m-1}$. This map can be viewed as

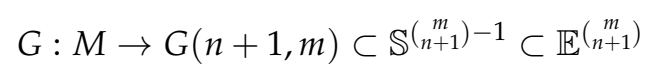

by considering the norm of vectors. We now define the pointwise 1-type spherical Gauss map of the spherical submanifold.

Definition 1. An oriented n-dimensional submanifold $M$ of $\mathbb{S}^{m-1}$ is said to have pointwise 1-type spherical Gauss map $G$ if it satisfies the partial differential equation

$$
\Delta G=f(G+C)
$$

for a nonzero smooth function $f$ on $M$ and some constant vector $C$. In particular, if $C$ is zero, the spherical Gauss map $G$ is said to be pointwise 1-type of the first kind. Otherwise, it is said to be of the second kind.

\section{Ruled Surfaces in $\mathbb{S}^{3}$ with Harmonic Spherical Gauss Maps}

Let $M$ be a ruled surface in the sphere $\mathbb{S}^{3}\left(\subset \mathbb{E}^{4}\right)$. Then, it is foliated by geodesics of $\mathbb{S}^{3}$ along a spherical curve. So, we can put its parametrization with spherical curves $\alpha=\alpha(s)$ and $\beta=\beta(s)$ by

$$
x=x(s, t)=\cos t \alpha(s)+\sin t \beta(s), s \in I, t \in J,
$$

where $I$ and $J$ are some open intervals. Without loss of generality, we may assume that

$$
\langle\alpha, \alpha\rangle=\langle\beta, \beta\rangle=\left\langle\alpha^{\prime}, \alpha^{\prime}\right\rangle=1 \text { and }\langle\alpha, \beta\rangle=\left\langle\alpha^{\prime}, \beta\right\rangle=0 .
$$

From now on, we always assume that the Parametrization (2) satisfies Condition (3) unless otherwise stated. Then, the spherical Gauss map $G$ of $M$ is given by

$$
G=\frac{x \wedge x_{s} \wedge x_{t}}{\left\|x \wedge x_{s} \wedge x_{t}\right\|}=\frac{1}{\sqrt{q}}\left(\cos t \alpha(s) \wedge \alpha^{\prime}(s) \wedge \beta(s)+\sin t \alpha(s) \wedge \beta^{\prime}(s) \wedge \beta(s)\right),
$$


where the function $q=q(s, t)$ is defined by

$$
q=\left\langle x_{s}, x_{s}\right\rangle=\cos ^{2} t+2 u(s) \cos t \sin t+w(s) \sin ^{2} t,
$$

where $u(s)=\left\langle\alpha^{\prime}, \beta^{\prime}\right\rangle$ and $w(s)=\left\langle\beta^{\prime}, \beta^{\prime}\right\rangle$ are functions of $s$.

By the definition of the Laplace operator $\Delta$, we have

$$
\begin{aligned}
\Delta G= & -q^{-\frac{7}{2}}\left(q_{s}\right)^{2}(\cos t A+\sin t B)+\frac{3}{2} q^{-\frac{5}{2}} q_{s}\left(\cos t A^{\prime}+\sin t B^{\prime}\right) \\
& +\frac{1}{2} q^{-\frac{5}{2}} q_{s s}(\cos t A+\sin t B)-q^{-\frac{3}{2}}\left(\cos t A^{\prime \prime}+\sin t B^{\prime \prime}\right) \\
& -\frac{1}{2} q^{-\frac{5}{2}}\left(q_{t}\right)^{2}(\cos t A+\sin t B)+\frac{1}{2} q^{-\frac{3}{2}} q_{t}(-\sin t A+\cos t B) \\
& +\frac{1}{2} q^{-\frac{3}{2}} q_{t t}(\cos t A+\sin t B)+q^{-\frac{1}{2}}(\cos t A+\sin t B),
\end{aligned}
$$

where we have put

$$
A=A(s)=\alpha(s) \wedge \alpha^{\prime}(s) \wedge \beta(s) \text { and } B=B(s)=\alpha(s) \wedge \beta^{\prime}(s) \wedge \beta(s) .
$$

On the other hand, we note that the vector fields $\alpha(s), \beta(s)$ and $\alpha^{\prime}(s)$ are mutually orthogonal for all $s$. Therefore, we can choose another unit vector field $\gamma(s)$ along the base curve $\alpha$ which forms an orthonormal frame in $\mathbb{E}^{4}$ together with $\alpha(s), \beta(s)$ and $\alpha^{\prime}(s)$.

Since $\Lambda^{3} \mathbb{E}^{4}$ is naturally identified with $\mathbb{E}^{4}$, we can define the inner product $X_{1} \wedge X_{2} \wedge X_{3}$ with $X_{4}$ as follows

$$
\ll X_{1} \wedge X_{2} \wedge X_{3}, X_{4} \gg=\operatorname{det}\left(\begin{array}{c}
X_{4} \\
X_{1} \\
X_{2} \\
X_{3}
\end{array}\right),
$$

where the determinant is taken by the $4 \times 4$ matrix made up of the components of the vectors $X_{1}, X_{2}$, $X_{3}, X_{4}$ in $\mathbb{E}^{4}$. Using this inner product, the vector field $A$ is represented by

$$
A=-\alpha \wedge \beta \wedge \alpha^{\prime}=-\ll \alpha \wedge \beta \wedge \alpha^{\prime}, \gamma(s) \gg \gamma(s)=-\gamma(s)
$$

by considering the orientation and the lengths of vectors. Similarly, we also have

$$
\begin{aligned}
& \ll\left(\alpha \wedge \beta \wedge \alpha^{\prime}\right)(s), \gamma(s) \gg=1, \\
& \ll(\alpha \wedge \beta \wedge \gamma)(s), \alpha^{\prime}(s) \gg=-1, \\
& \ll\left(\alpha \wedge \alpha^{\prime} \wedge \gamma\right)(s), \beta(s) \gg=1, \\
& \ll\left(\beta \wedge \alpha^{\prime} \wedge \gamma\right)(s), \alpha(s) \gg=-1
\end{aligned}
$$

for all s. By virtue of (7), we can obtain the following

$$
\begin{aligned}
& \left(\begin{array}{rl}
A= & =\gamma \\
A^{\prime} & =b \beta+\varphi \alpha^{\prime} \\
A^{\prime \prime}= & -\varphi \alpha+\left(b^{\prime}-u \varphi\right) \beta+\left(u b+\varphi^{\prime}\right) \alpha^{\prime}+\left(b^{2}+\varphi^{2}\right) \gamma
\end{array}\right. \\
& \left(\begin{array}{rl}
B= & b \alpha^{\prime}-u \gamma \\
B^{\prime}= & -b \alpha+\left(b^{\prime}+u \varphi\right) \alpha^{\prime}+\left(b \varphi-u^{\prime}\right) \gamma \\
B^{\prime \prime}= & -\left(2 b^{\prime}+u \varphi\right) \alpha+\left(u^{\prime} b-u b^{\prime}-u^{2} \varphi-b^{2} \varphi\right) \beta \\
& +\left(b^{\prime \prime}+2 u^{\prime} \varphi+u \varphi^{\prime}-b-b \varphi^{2}\right) \alpha^{\prime}+\left(2 b^{\prime} \varphi+u \varphi^{2}+b \varphi^{\prime}-u^{\prime \prime}\right) \gamma
\end{array}\right.
\end{aligned}
$$


which imply that the spherical Gauss map $G$ represented by (4) reduces to

$$
G=\frac{1}{\sqrt{q}}\left((b \sin t) \alpha^{\prime}-(\cos t+u \sin t) \gamma\right)
$$

where we have put $b=b(s)=\left\langle\beta^{\prime}(s), \gamma(s)\right\rangle$ and $\varphi=\varphi(s)=\left\langle\alpha^{\prime \prime}(s), \gamma(s)\right\rangle$.

Theorem 1. Let $M$ be a ruled surface in the sphere $\mathbb{S}^{3}$. Then, $M$ has a harmonic spherical Gauss map if and only if $M$ is totally geodesic in $\mathbb{S}^{3}$.

Proof. Suppose that the spherical Gauss map $G$ is harmonic, i.e., $\Delta G=\mathbf{0}$, where $\mathbf{0}$ denotes zero vector. Then, (6) implies

$$
\begin{gathered}
\left\{-\left(q_{s}\right)^{2}+\frac{1}{2} q q_{s s}-\frac{1}{2} q\left(q_{t}\right)^{2}+\frac{1}{2} q^{2} q_{t t}+q^{3}\right\}(\cos t A+\sin t B) \\
+\frac{3}{2} q q_{s}\left(\cos t A^{\prime}+\sin t B^{\prime}\right)-q^{2}\left(\cos t A^{\prime \prime}+\sin t B^{\prime \prime}\right)+\frac{1}{2} q^{2} q_{t}(-\sin t A+\cos t B)=\mathbf{0} .
\end{gathered}
$$

By the orthogonality of vector fields $\alpha, \beta, \alpha^{\prime}$ and $\gamma$, putting (8) into (10) gives us

$$
\begin{gathered}
\frac{3}{2} q q_{s} b \sin t-q^{2} \varphi \cos t-q^{2}\left(2 b^{\prime}+u \varphi\right) \sin t=0, \\
\frac{3}{2} q q_{s} b \cos t-q^{2}\left(b^{\prime}-u \varphi\right) \cos t-q^{2}\left(u^{\prime} b-u b^{\prime}-u^{2} \varphi-b^{2} \varphi\right) \sin t=0
\end{gathered}
$$

as the coefficients of the vectors $\alpha$ and $\beta$, respectively. Using the equation for $q$ of (5) and the fact that $q>0,(11)$ and (12) can be expressed as

$$
\begin{array}{r}
-\varphi \cos ^{3} t+\left(-3 u \varphi-2 b^{\prime}\right) \cos ^{2} t \sin t \\
+\left(3 u^{\prime} b-4 u b^{\prime}-3 u^{2} \varphi-b^{2} \varphi\right) \cos t \sin ^{2} t \\
+\left(3 u u^{\prime} b+b^{2} b^{\prime}-2 u^{2} b^{\prime}-u^{3} \varphi-u b^{2} \varphi\right) \sin ^{3} t=0
\end{array}
$$

and

$$
\begin{array}{r}
\left(u \varphi-b^{\prime}\right) \cos ^{3} t+\left(2 u^{\prime} b+3 u^{2} \varphi-u b^{\prime}+b^{2} \varphi\right) \cos ^{2} t \sin t \\
+\left(u u^{\prime} b+2 b^{2} b^{\prime}+3 u^{3} \varphi+3 u b^{2} \varphi+u^{2} b^{\prime}\right) \cos t \sin ^{2} t \\
+\left(u^{4} \varphi+b^{4} \varphi+u^{3} b^{\prime}-u^{2} u^{\prime} b+2 u^{2} b^{2} \varphi+u b^{2} b^{\prime}-u^{\prime} b^{3}\right) \sin ^{3} t=0,
\end{array}
$$

respectively. We easily see that the trigonometric functions of $t$ of (13) and (14) are linearly independent for all $t$. Therefore, we can see that

$$
\varphi=0 \quad \text { and } \quad b^{\prime}=0
$$

by considering the coefficients of the terms containing ' $\cos ^{3} t$ ' of (13) and (14), respectively. From the coefficients of the term containing ' $\cos t \sin ^{2} t$ ' of (13), we get

$$
u^{\prime} b=0 .
$$

Suppose that $b$ is a nonzero constant on $M$. Then, (11) and (16) imply $q_{s}=0$. Putting it into (10) yields

$$
\begin{gathered}
\left\{-\frac{1}{2}\left(q_{t}\right)^{2}+\frac{1}{2} q q_{t t}+q^{2}\right\}(\cos t A+\sin t B) \\
-q\left(\cos t A^{\prime \prime}+\sin t B^{\prime \prime}\right)+\frac{1}{2} q q_{t}(-\sin t A+\cos t B)=\mathbf{0} .
\end{gathered}
$$


In this case, the vectors are reduced to

$$
\left\{\begin{array}{l}
A=-\gamma \\
A^{\prime \prime}=u b \alpha^{\prime}+b^{2} \gamma \\
B=b \alpha^{\prime}-u \gamma \\
B^{\prime \prime}=-b \alpha^{\prime}
\end{array}\right.
$$

Using (18), we note that Equation (17) can be regarded as the form of the linear combination of two orthogonal vectors $\alpha^{\prime}$ and $\gamma$ with trigonometric functions in $t$ as coefficients. By a straightforward computation, we can see that the coefficient of $\gamma$ of (17) is given by

$$
\begin{aligned}
-2 b^{2}\left(\cos ^{5} t+\right. & \left.u \cos ^{4} t \sin t+2 \cos ^{3} t \sin ^{2} t+2 u \cos ^{2} t \sin ^{3} t+\cos t \sin ^{4} t+u \sin ^{5} t\right) \\
& =-2 b^{2}(\cos t+u \sin t)\left(\cos ^{2} t+\sin ^{2} t\right)^{2} \\
& =-2 b^{2}(\cos t+u \sin t)=0
\end{aligned}
$$

which implies that $b=0$, a contradiction to $b \neq 0$. Therefore, the constant $b$ is zero. With the help of (15), we get from (8) that

$$
A^{\prime}=\mathbf{0} \text { and } B=u A .
$$

Since the spherical Gauss map $G=-\frac{1}{\sqrt{q}}(\cos t+u \sin t) \gamma$ is a unit normal vector field of the ruled surface $M$ to the unit sphere $\mathbb{S}^{3}$, it is easily obtained that the shape operator $S$ of $M$ in $\mathbb{S}^{3}$ vanishes, i.e., $M$ is totally geodesic in $\mathbb{S}^{3}$.

Conversely, if $M$ is a totally geodesic surface of $\mathbb{S}^{3}$, i.e., $M$ is a great sphere of $\mathbb{S}^{3}$, it is not hard to show that the spherical Gauss map of $M$ is harmonic. It completes the proof.

\section{A Ruled Surface in $\mathbb{S}^{3}$ with a Finite-Type Spherical Gauss Map}

In this section, we will investigate a ruled surface $M$ in $\mathbb{S}^{3}$ parameterized by (2) with a finite-type spherical Gauss map.

Using (6), (8) and (9), the Laplacian $\Delta G$ can be put as

$$
\Delta G=-\frac{\left(q_{s}\right)^{2}}{q^{\frac{7}{2}}}\left((b \sin t) \alpha^{\prime}-(\cos t+u \sin t) \gamma\right)+\frac{1}{q^{\frac{5}{2}}} P_{1}(s, t),
$$

where $P_{1}$ is a vector field formed with the linear combination of the orthogonal vector fields $\alpha, \beta, \alpha^{\prime}$ and $\gamma$ together with the coefficients of trigonometric functions in $t$ and functions in $s$. Proceeding by induction, we get

$$
\Delta^{m} G=a_{m} \frac{\left(q_{s}\right)^{2 m}}{q^{3 m+\frac{1}{2}}}\left((b \sin t) \alpha^{\prime}-(\cos t+u \sin t) \gamma\right)+\frac{1}{q^{3 m-\frac{1}{2}}} P_{m}(s, t)
$$

for any positive integer $m$, where $P_{m}$ is a vector field formed with the linear combination of the orthogonal vector fields $\alpha, \beta, \alpha^{\prime}$ and $\gamma$ together with the coefficients of trigonometric functions in $t$ and functions in $s$, and $a_{m}$ is a nonzero constant satisfying $a_{m}=(3 m-1)\left(\frac{5}{2}-3 m\right) a_{m-1}$ with $a_{0}=1$.

Suppose that the spherical Gauss map $G$ of $M$ is of finite-type. Then, we have

$$
\Delta^{k} G+c_{1} \Delta^{k-1} G+c_{2} \Delta^{k-2} G+\cdots+c_{k-1} \Delta G=\mathbf{0}
$$

for some constants $c_{1}, c_{2}, \ldots, c_{k-1} \in \mathbb{R}$ and a positive integer $k$. By the orthogonality of the vectors $\alpha, \beta$, $\alpha^{\prime}$ and $\gamma$, substituting (19) into (20) gives us the coefficients of $\alpha^{\prime}$ and $\gamma$ as follows

$$
\left(q_{s}\right)^{2 k} b \sin t=q F_{1}(s, t)
$$


and

$$
\left(q_{s}\right)^{2 k}(\cos t+u \sin t)=q F_{2}(s, t)
$$

respectively, from which,

$$
\begin{array}{r}
\left(2 u^{\prime} \cos t \sin t+w^{\prime} \sin ^{2} t\right)^{2 k}(\cos t+(u+b) \sin t) \\
=\left(\cos ^{2} t+2 u \cos t \sin t+w \sin ^{2} t\right) F(s, t)
\end{array}
$$

for some polynomials $F_{1}$ and $F_{2}$ in ' $\cos t^{\prime}$ and 'sin $t$ ' with functions of $s$ as coefficients, where $F(s, t)=$ $F_{1}(s, t)+F_{2}(s, t)$.

By the linear independence of the trigonometric functions $\cos ^{2} t, \cos t \sin t$ and $\sin ^{2} t$, we may put

$$
F(s, t)=\left(q_{s}\right)^{l} R(s, t)=\left(2 u^{\prime} \cos t \sin t+w^{\prime} \sin ^{2} t\right)^{l} R(s, t),
$$

where $l$ is a non-negative integer less than $2 k$ and $R(s, t)$ is some polynomial in ' $\cos ^{n-k} t \sin ^{k} t^{\prime}$, $k=0,1, \ldots, n$, with functions in $s$ as coefficients such that $R(s, t)$ and $q_{s}$ are relatively prime. That is, $R(s, t)$ is of the form

$$
R(s, t)=\sum_{k=0}^{n} \Gamma_{k}(s) \cos ^{n-k} t \sin ^{k} t
$$

for some functions $\Gamma_{k}$ in $s$. Here, the degree of $R(s, t)$ is $n$. Then, (21) becomes

$$
\begin{gathered}
\left(2 u^{\prime} \cos t \sin t+w^{\prime} \sin ^{2} t\right)^{2 k-l}(\cos t+(u+b) \sin t) \\
=\left(\cos ^{2} t+2 u \cos t \sin t+w \sin ^{2} t\right) R(s, t) .
\end{gathered}
$$

By putting $\theta=\tan t$ in (22), we get

$$
\begin{aligned}
& \left(\frac{2 u^{\prime} \theta}{\left(1+\theta^{2}\right)}+\frac{w^{\prime} \theta^{2}}{\left(1+\theta^{2}\right)}\right)^{2 k-l}\left(\frac{1}{\sqrt{1+\theta^{2}}}+\frac{(u+b) \theta}{\sqrt{1+\theta^{2}}}\right) \\
& =\left(\frac{1}{\left(1+\theta^{2}\right)}+\frac{2 u \theta}{\left(1+\theta^{2}\right)}+\frac{w \theta^{2}}{\left(1+\theta^{2}\right)}\right) \frac{1}{\left(\sqrt{1+\theta^{2}}\right)^{n}} \bar{R}(s, \theta),
\end{aligned}
$$

or, equivalently,

$$
\begin{aligned}
& \left(2 u^{\prime} \theta+w^{\prime} \theta^{2}\right)^{2 k-l}\left(1+\theta^{2}\right)^{\frac{n}{2}+1}(1+(u+b) \theta) \\
& =\left(1+2 u \theta+w \theta^{2}\right)\left(1+\theta^{2}\right)^{2 k-l+\frac{1}{2}} \bar{R}(s, \theta),
\end{aligned}
$$

where $\bar{R}(s, \theta)$ is a polynomial in $\theta$ with functions in $s$ as coefficients such that

$$
\bar{R}(s, \theta)=\left(\sqrt{1+\theta^{2}}\right)^{n} R(s, t(\theta)) .
$$

We note that two polynomials $\left(2 u^{\prime} \theta+w^{\prime} \theta^{2}\right)$ and $\bar{R}(s, \theta)$ are relatively prime, where the former one is obtained from $q_{s}$.

Now, we will deal with possible cases derived from (23). Considering the degree of (23) with respect to $\theta$ and the linear independence of $\left(2 u^{\prime} \theta+w^{\prime} \theta^{2}\right)$ and $\bar{R}(s, \theta)$, we can put

$$
\left(2 u^{\prime} \theta+w^{\prime} \theta^{2}\right)^{2 k-l}=\lambda(s)\left(1+2 u \theta+w \theta^{2}\right)\left(1+\theta^{2}\right)^{2 k-l-1},
$$

or,

$$
\left(2 u^{\prime} \theta+w^{\prime} \theta^{2}\right)^{2 k-l}\left(1+\theta^{2}\right)=\lambda(s)\left(1+2 u \theta+w \theta^{2}\right)\left(1+\theta^{2}\right)^{2 k-l}
$$


for some function $\lambda$ in $s$.

Recall that (24) is a polynomial in $\theta$. So, by comparing the smallest power of both sides of (24) with respect to $\theta$, we can see that ' $2 k-l$ ' must be zero. Therefore, (24) becomes of the form

$$
\left(1+\theta^{2}\right)=\lambda(s)\left(1+2 u \theta+w \theta^{2}\right) .
$$

It follows that

$$
u=0 \quad \text { and } \quad w=1,
$$

from which, we get the function $q$ is constant with value 1 and the metric tensor $g$ of $M$ is given by

$$
g=\left(\begin{array}{ll}
1 & 0 \\
0 & 1
\end{array}\right),
$$

from which, we see that $M$ is flat in $\mathbb{E}^{4}$. It also gives us $\Delta x=2 x$ and hence $M$ is minimal in $\mathbb{S}^{3}$. Therefore, $M$ is one of the isoparametric surfaces in $\mathbb{S}^{3}$, which is the Clifford torus $\mathbb{S}^{1}(1 / \sqrt{2}) \times \mathbb{S}^{1}(1 / \sqrt{2})$.

Together with Theorem 1, we have

Theorem 2. Let $M$ be a complete ruled surface in the sphere $\mathbb{S}^{3}$ with finite-type spherical Gauss map. Then, $M$ is either the Clifford torus $\mathbb{S}^{1}(1 / \sqrt{2}) \times \mathbb{S}^{1}(1 / \sqrt{2})$ or a totally geodesic surface in $\mathbb{S}^{3}$.

Corollary 1. Let $M$ be a ruled surface in the sphere $\mathbb{S}^{3}$. If the spherical Gauss map $G$ of $M$ is of finite-type, then both $M$ and $G$ are of 1-type. In particular, $\Delta x=2 x$ and either $\Delta G=\mathbf{0}$ or $\Delta G=2 G$.

\section{Ruled Surfaces in $\mathbb{S}^{3}$ with Pointwise 1-Type Spherical Gauss Maps of the First Kind}

In this section, we will study a ruled surface in $\mathbb{S}^{3}$ with pointwise 1-type spherical Gauss map $G$ of the first kind, i.e., $\Delta G=f G$ for some nonzero smooth function $f$. Let $M$ be a ruled surface in the sphere $\mathbb{S}^{3}\left(\subset \mathbb{E}^{4}\right)$ parameterized by (2). Then, using (6), equation $\Delta G=f G$ gives

$$
\begin{gathered}
\left\{-\left(q_{s}\right)^{2}+\frac{1}{2} q q_{s s}-\frac{1}{2} q\left(q_{t}\right)^{2}+\frac{1}{2} q^{2} q_{t t}+(1-f) q^{3}\right\}(\cos t A+\sin t B) \\
+\frac{3}{2} q q_{s}\left(\cos t A^{\prime}+\sin t B^{\prime}\right)-q^{2}\left(\cos t A^{\prime \prime}+\sin t B^{\prime \prime}\right)+\frac{1}{2} q^{2} q_{t}(-\sin t A+\cos t B)=\mathbf{0} .
\end{gathered}
$$

With the help of (8), by comparing two equations, (10) and (25), we can see that the coefficients of the vectors $\alpha$ and $\beta$ of (25) coincide with those of $\alpha$ and $\beta$ of (10). Therefore, we obtain (13) and (14), or, equivalently, we have

$$
\varphi=0, \quad b^{\prime}=0 \quad \text { and } \quad u^{\prime} b=0 .
$$

Similarly as we did to the constant $b$ in Section 3, we will show the constant $b$ is nonzero and hence $u$ is a constant. Suppose that $b=0$ on $M . \Delta G=f G$ with $b=0$ gives

$$
\begin{aligned}
\left\{-\left(q_{s}\right)^{2}+\right. & \left.\frac{1}{2} q q_{s s}-\frac{1}{2} q\left(q_{t}\right)^{2}+\frac{1}{2} q^{2} q_{t t}+(1-f) q^{3}\right\}(-\cos t-u \sin t) \\
& +\frac{3}{2} q q_{s}\left(-u^{\prime} \sin t\right)-q^{2}\left(-u^{\prime \prime} \sin t\right)+\frac{1}{2} q^{2} q_{t}(\sin t-u \cos t)=0
\end{aligned}
$$

from which,

$$
f(\cos t+u \sin t)^{7}=0 .
$$


It implies that $f$ is vanishing. It is a contradiction and thus we conclude that $b$ is nonzero. Then, we have $q_{s}=0$. With the help of (18), (25) is reduced to

$$
\begin{aligned}
& \left\{-\frac{1}{2}\left(q_{t}\right)^{2}+\frac{1}{2} q q_{t t}+(1-f) q^{2}\right\}(\cos t A+\sin t B) \\
& -q\left(\cos t A^{\prime \prime}+\sin t B^{\prime \prime}\right)+\frac{1}{2} q q_{t}(-\sin t A+\cos t B)=\mathbf{0}
\end{aligned}
$$

which provides us with

$$
f q^{2} \sin t=\left\{-\frac{1}{2}\left(q_{t}\right)^{2}+\frac{1}{2} q q_{t t}+q^{2}+q\right\} \sin t+\left(\frac{1}{2} q q_{t}-u q\right) \cos t
$$

as the coefficients of the vector $\alpha^{\prime}$.

We note that $w=u^{2}+b^{2}$. By a straightforward computation, we get

$$
f=\frac{2 b^{2}}{q^{2}}
$$

Consequently, if a ruled surface $M$ has pointwise 1-type spherical Gauss map of the first kind, we see that the constant $b$ is nonzero and $\varphi=0$, that is, the curves $\alpha$ and $\beta$ satisfy

$$
\alpha^{\prime} \wedge \beta^{\prime} \neq \mathbf{0} \quad \text { and } \quad \alpha^{\prime \prime} \wedge \alpha \wedge \beta=\mathbf{0}
$$

for all $s$. Now, we consider the curve $\delta(s)$ on the sphere $\mathbb{S}^{3}\left(\sqrt{\frac{u^{2}+1}{b^{2}}}\right)$ given by

$$
\delta(s)=-\frac{u}{b} \alpha(s)+\frac{1}{b} \beta(s) .
$$

We note that the curve $\delta(s)$ is an integral curve of $\gamma$, that is, $\delta^{\prime}=\gamma$. Then, we can easily show that the spherical Gauss map $G$ of a ruled surface $M$ in $\mathbb{S}^{3}$ parameterized by

$$
\begin{aligned}
M: x(s, t) & =\alpha(s) \cos t+\beta(s) \sin t \\
& =(\cos t+u \sin t) \alpha(s)+b \sin t \delta(s)
\end{aligned}
$$

is of pointwise 1-type of the first kind. Indeed, it follows that

$$
\Delta G=\left(\frac{2 b^{2}}{q^{2}}\right) G
$$

Therefore, we have

Theorem 3. Let $M$ be a ruled surface in the unit sphere $\mathbb{S}^{3}$. If $M$ has pointwise 1-type spherical Gauss map of the first kind, then $M$ is part of the ruled surface in $\mathbb{S}^{3}$ parameterized by (27) satisfying (26).

Example 1. The curves $\alpha(s)$ and $\delta(s)$, given by

$$
\alpha(s)=\left(\frac{1}{\sqrt{2}} \cos s, \frac{1}{\sqrt{2}} \sin s, \frac{1}{\sqrt{2}} \cos s, \frac{1}{\sqrt{2}} \sin s\right)
$$

and

$$
\delta(s)=\left(\frac{1}{2 \sqrt{2}} \sin (2 s),-\frac{1}{2 \sqrt{2}} \cos (2 s),-\frac{1}{2 \sqrt{2}} \sin (2 s), \frac{1}{2 \sqrt{2}} \cos (2 s)\right)
$$


are unit speed curves on the sphere $\mathbb{S}^{3}$ and the sphere $\mathbb{S}^{3}\left(\frac{1}{2}\right)$, respectively. In this case, it is clear that $u=0$ and $b=2$. Then, the ruled surface $M$ in the sphere $\mathbb{S}^{3}$ defined by

$$
\begin{aligned}
M: x(s, t)= & \alpha(s) \cos t+2 \delta(s) \sin t \\
= & \frac{1}{\sqrt{2}}(\cos t \cos s+\sin t \sin (2 s), \cos t \sin s-\sin t \cos (2 s), \\
& \quad \cos t \cos s-\sin t \sin (2 s), \cos t \sin s+\sin t \cos (2 s))
\end{aligned}
$$

has pointwise 1-type spherical Gauss map G of the first kind

$$
\Delta G=\frac{8}{\left(\cos ^{2} t+4 \sin ^{2} t\right)^{2}} G
$$

\section{Ruled Surfaces in $\mathbb{S}^{3}$ with Pointwise 1-Type Spherical Gauss Maps of the Second Kind}

In this section, we will investigate a ruled surface $M$ in $\mathbb{S}^{3}$ parameterized by (2) with a pointwise 1-type spherical Gauss map of the second kind, that is, the spherical Gauss map $G$ of $M$ satisfies

$$
\Delta G=f(G+\mathbf{C})
$$

for some nonzero function $f$ of $s$ and $t$ and a non-zero constant vector $\mathbf{C}$. If we consider a non-empty open subset $U=\{(s, t) \in I \times J \mid f(s, t) \neq 0\}$, then we can put

$$
\mathbf{C}=\frac{\Delta G-f G}{f}
$$

which yields that

$$
f(\Delta G-f G)_{t}=f_{t}(\Delta G-f G)
$$

on $U$.

Now, we consider the open subset $U_{0}=\left\{(s, t) \in U \mid f_{t}(s, t) \neq 0\right\}$ and suppose that $U_{0}$ is non-empty. With the help of (8) and (25), we can get from (29),

$$
f\left(q^{-\frac{7}{2}} P\right)_{t}=f_{t}\left(q^{-\frac{7}{2}} P\right) \quad \text { and } \quad f\left(q^{-\frac{7}{2}} Q\right)_{t}=f_{t}\left(q^{-\frac{7}{2}} Q\right),
$$

or, equivalently,

$$
f\left(-\frac{7}{2} q_{t} P+q P_{t}\right)=q f_{t} P \quad \text { and } \quad f\left(-\frac{7}{2} q_{t} Q+q Q_{t}\right)=q f_{t} Q
$$

as the coefficients of the vectors $\alpha$ and $\beta$ of (29), respectively, where we have put

$$
P(s, t)=\frac{3}{2} q q_{s} b \sin t-q^{2} \varphi \cos t-q^{2}\left(u \varphi+2 b^{\prime}\right) \sin t
$$

and

$$
Q(s, t)=\frac{3}{2} q q_{s} b \cos t+q^{2}\left(u \varphi-b^{\prime}\right) \cos t-q^{2}\left(u^{\prime} b-u b^{\prime}-u^{2} \varphi-b^{2} \varphi\right) \sin t
$$

Now, we will consider a few lemmas to reach a conclusion for this section. 
Lemma 1. Let $M$ be a ruled surface in the unit sphere $\mathbb{S}^{3}$ parameterized by (2) with a pointwise 1-type spherical Gauss map of the second kind. If $U_{0}=\left\{(s, t) \in I \times J \mid f_{t}(s, t) \neq 0\right\}(\subset U)$ is non-empty, then

$$
\alpha^{\prime} \wedge \beta^{\prime}=\mathbf{0} \text { on } U_{0} .
$$

Proof. We suppose that the function $b(s)$ is non-vanishing on some open set $U_{1}$ of $U_{0}$. We first consider the case that at least one of two equations $P(s, t)$ and $Q(s, t)$ is vanishing on some subset of $U_{1}$, say $P(s, t)=0$. Then, we can easily show that

$$
\varphi=0 \quad \text { and } \quad b^{\prime}=0
$$

by considering the linear independence of the trigonometric functions of (31). Since $b$ is a nonzero constant, (31) and (33) imply $q_{s}=0$. Thus, the function $Q(s, t)$ of (32) has to be identically zero on that subset $U_{1}$. Similarly, if $Q(s, t)=0$, we can derive $P(s, t)=0$. Therefore, we suppose that both $\Phi(s, t)$ and $\Psi(s, t)$ are identically zero on $U_{1}$. In this case, Equation (29) can be put as

$$
\left(f_{t} q^{-\frac{5}{2}} \Lambda_{1}\right) \alpha^{\prime}+\left(f_{t} q^{-\frac{5}{2}} \Lambda_{2}\right) \gamma=\left(f\left(q^{-\frac{5}{2}} \Lambda_{1}\right)_{t}\right) \alpha^{\prime}+\left(f\left(q^{-\frac{5}{2}} \Lambda_{2}\right)_{t}\right) \gamma
$$

which yields that

$$
\frac{f_{t}}{f}=\frac{\left(q^{-\frac{5}{2}} \Lambda_{1}\right)_{t}}{q^{-\frac{5}{2}} \Lambda_{1}}=\frac{\left(q^{-\frac{5}{2}} \Lambda_{2}\right)_{t}}{q^{-\frac{5}{2}} \Lambda_{2}}
$$

by comparing the coefficients of two orthogonal vectors $\alpha^{\prime}$ and $\gamma$, where we have put

$$
\Lambda_{1}(s, t)=\left\{-\frac{1}{2}\left(q_{t}\right)^{2}+\frac{1}{2} q q_{t t}+(1-f) q_{2}\right\} b \sin t-q b(u \cos t-\sin t)+\frac{1}{2} q q_{t} b \cos t
$$

and

$$
\begin{aligned}
\Lambda_{2}(s, t)= & \left\{\frac{1}{2}\left(q_{t}\right)^{2}-\frac{1}{2} q q_{t t}-(1-f) q_{2}\right\}(\cos t+u \sin t) \\
& -q b^{2} \cos t+\frac{1}{2} q q_{t}(\sin t-u \cos t) .
\end{aligned}
$$

By taking the integration to (34) with respect to $t$, we see that the function $f$ takes the form

$$
f=y_{1}(s)\left(q^{-\frac{5}{2}} \Lambda_{1}\right)=y_{2}(s)\left(q^{-\frac{5}{2}} \Lambda_{2}\right)
$$

for some non-vanishing functions $y_{1}$ and $y_{2}$ of $s$. If we put (35) and (36) into (37), then we can obtain the formulas for $f$ as

$$
f=\frac{2 b^{3} y_{1}(s) \sin t}{q^{2}\left(q^{\frac{1}{2}}+b y_{1}(s) \sin t\right)}=\frac{-2 b^{2} y_{2}(s)(\cos t+u \sin t)}{q^{2}\left(q^{\frac{1}{2}}-y_{2}(s)(\cos t+u \sin t)\right)}
$$

Comparing the last two equations in (38), we get

$$
\left(y_{2} \cos t+\left(b y_{1}+u y_{2}\right) \sin t\right) q^{\frac{1}{2}}=0
$$

which implies

$$
y_{2}(s)=0 \text { and } y_{1}(s)=0
$$


because of $q \neq 0$, but it contradicts $f \neq 0$. Consequently, this case never occurs. Therefore, we may assume that both $P(s, t)$ and $Q(s, t)$ are both non-vanishing on $U_{1}\left(\subset U_{0}\right)$. Then, equations of (30) give

$$
\frac{f_{t}}{f}=\frac{\left(q^{-\frac{7}{2}} P\right)_{t}}{\left(q^{-\frac{7}{2}} P\right)}=\frac{\left(q^{-\frac{7}{2}} Q\right)_{t}}{\left(q^{-\frac{7}{2}} Q\right)}
$$

on $U_{1}$ and thus the function $f$ is of the form

$$
f=g_{1}(s) q^{-\frac{7}{2}} P(s, t)=g_{2}(s) q^{-\frac{7}{2}} Q(s, t)
$$

which implies

$$
\begin{gathered}
g_{1}(s)\left\{\frac{3}{2} b\left(2 u^{\prime} \cos t \sin ^{2} t+w^{\prime} \sin ^{3} t\right)-\varphi\left(\cos ^{3} t+2 u \cos ^{2} t \sin t+w \cos t \sin ^{2} t\right)\right. \\
\left.-\left(u \varphi-2 b^{\prime}\right)\left(\cos ^{2} t \sin t+2 u \cos t \sin ^{2} t+w \sin ^{3} t\right)\right\} \\
=g_{2}(s)\left\{\frac{3}{2} b\left(2 u^{\prime} \cos ^{2} t \sin t+w^{\prime} \cos t \sin ^{2} t\right)\right. \\
+\left(u \varphi-b^{\prime}\right)\left(\cos ^{3} t+2 u \cos ^{2} t \sin t+w \cos t \sin ^{2} t\right) \\
\left.-\left(u^{\prime} b-u b^{\prime}-u^{2} \varphi-b^{2} \varphi\right)\left(\cos ^{2} t \sin t+2 u \cos t \sin ^{2} t+w \sin ^{3} t\right)\right\}
\end{gathered}
$$

for some non-vanishing functions $g_{1}$ and $g_{2}$ of $s$ on $U_{1}$ because of $q>0$. By the linear independence of trigonometric functions $\cos ^{3-k} t \sin ^{k} t$ of (41) for $k=0, \ldots, 3$, we have

$$
\begin{gathered}
g_{1} \varphi=g_{2}\left(b^{\prime}-u \varphi\right), \\
g_{1}\left(-3 u \varphi-2 b^{\prime}\right)=g_{2}\left(2 u^{\prime} b+3 u^{2} \varphi-u b^{\prime}+b^{2} \varphi\right), \\
g_{1}\left(3 u^{\prime} b-4 u b^{\prime}-3 u^{2} \varphi-b^{2} \varphi\right)=g_{2}\left(u u^{\prime} b+2 b^{2} b^{\prime}+3 u^{3} \varphi+3 u b^{2} \varphi+u^{2} b^{\prime}\right)
\end{gathered}
$$

and

$$
\begin{gathered}
g_{1}\left(3 u u^{\prime} b+b^{2} b^{\prime}-2 u^{2} b^{\prime}-u^{3} \varphi-u b^{2} \varphi\right) \\
=g_{2}\left(u^{4} \varphi+b^{4} \varphi+u^{3} b^{\prime}-u^{2} u^{\prime} b+2 u^{2} b^{2} \varphi+u b^{2} b^{\prime}-u^{\prime} b^{3}\right)
\end{gathered}
$$

as the coefficients of terms containing ' $\cos ^{3} t^{\prime},{ }^{\prime} \cos ^{2} t \sin t^{\prime},{ }^{\prime} \cos t \sin ^{2} t^{\prime}$ and $\sin ^{3} t^{\prime}$, respectively. Substituting (42) into (43), we get

$$
-2 b^{\prime} g_{1}=g_{2}\left(2 u b^{\prime}+2 u^{\prime} b+b^{2} \varphi\right)
$$

which implies

$$
u^{\prime} b g_{1}=g_{2}\left(-u u^{\prime} b+b^{2} b^{\prime}\right)
$$

with the aid of (42) and (44). Finally, putting (42), (46) and (47) into (45) allows us to have

$$
\frac{3}{2} b^{4} \varphi g_{2}=0
$$

and hence $\varphi=0$ because $b$ and $g_{2}$ are non-vanishing on $U_{1}$. From (42) and (43), we can see that

$$
b^{\prime}=0 \quad \text { and } \quad u^{\prime}=0 \text {, }
$$


or, equivalently,

$$
q_{s}=0 \text { on } U_{1} \text {. }
$$

Since $\varphi=0$ and $q_{s}=0$ on $U_{1}$, the non-vanishing function $P(s, t)$ of (31) on $U_{0}$ becomes identically zero on $U_{1} \subset U_{0}$, a contradiction. Therefore, we conclude that the set $U_{1}$ is empty, which means that $\beta^{\prime}=u \alpha^{\prime}$ on $U_{0}$ as we desired.

Now, we will examine the set $x\left(U_{0}\right)$ of $\mathbb{S}^{3}$. In Lemma 1 , we showed that $b=\left\langle\beta^{\prime}, \gamma\right\rangle=0$ on $U_{0}$. Then, we have

$$
q=(\cos t+u(s) \sin t)^{2}
$$

and the spherical Gauss map $G$ of (9) is given by

$$
G=-\gamma
$$

From (41), we see that

$$
\left(\left(g_{1}+u g_{2}\right) \varphi\right)(s)=0 \text { on } U_{0} .
$$

If $\varphi=0$ on some subset $U_{2}$ of $U_{0}$ with $\operatorname{int}\left(U_{2}\right) \neq \varnothing$, then

$$
G^{\prime}=(-\gamma)^{\prime}=\varphi \alpha^{\prime}=\mathbf{0}
$$

which means that the spherical Gauss map $G$ is constant and thus $\Delta G=0$ on that subset. Since the spherical Gauss map is of pointwise 1-type of the second kind and $\mathrm{C}$ is a constant vector, $G=-$ C globally.

Now, we suppose that the function $\varphi$ is non-vanishing on $U_{0}$. From (49), we see that $\left(g_{1}+u g_{2}\right) \equiv 0$ on $U_{0}$ and then, the function $f$ of (40) is simplified as

$$
f(s, t)=-\frac{\varphi(s) g_{1}(s)}{(\cos t+u(s) \sin t)^{2}}
$$

so equation $\Delta G=f(G+\mathbf{C})$ can be expressed as follows

$$
\frac{u^{\prime} \sin t}{(\cos t+u \sin t)^{3}} \gamma^{\prime}-\frac{1}{(\cos t+u \sin t)^{2}} \gamma^{\prime \prime}=\frac{\varphi g_{1}}{(\cos t+u \sin t)^{2}}(-\gamma+\mathbf{C}) .
$$

With the help of (8), it follows that

$$
\begin{aligned}
u^{\prime} \varphi \sin t \alpha^{\prime} & +(\cos t+u \sin t)\left(\varphi \alpha+u \varphi \beta-\varphi^{\prime} \alpha^{\prime}-\varphi^{2} \gamma\right) \\
& =\varphi g_{1}(\cos t+u \sin t)(\gamma-\mathbf{C})
\end{aligned}
$$

which guarantees that

$$
\varphi \alpha+u \varphi \beta-\varphi^{\prime} \alpha^{\prime}-\varphi^{2} \gamma=\varphi g_{1}(\gamma-\mathbf{C})
$$

by considering the terms containing ' $\cos t^{\prime}$. Thus, the constant vector $\mathbf{C}$ can be put

$$
\mathbf{C}=-\frac{1}{g_{1}} \alpha-\frac{u}{g_{1}} \beta+\frac{\varphi^{\prime}}{\varphi g_{1}} \alpha^{\prime}+\left(\frac{\varphi}{g_{1}}+1\right) \gamma,
$$

from which,

$$
\begin{aligned}
\mathbf{0}= & -\left(\left(\frac{1}{g_{1}}\right)^{\prime}+\frac{\varphi^{\prime}}{\varphi g_{1}}\right) \alpha-\left(\left(\frac{u}{g_{1}}\right)^{\prime}+\frac{u \varphi^{\prime}}{\varphi g_{1}}\right) \beta \\
& +\left(-\frac{1}{g_{1}}-\frac{u^{2}}{g_{1}}+\left(\frac{\varphi^{\prime}}{\varphi g_{1}}\right)^{\prime}-\frac{\varphi^{2}}{g_{1}}-\varphi\right) \alpha^{\prime}+\left(\frac{\varphi^{\prime}}{g_{1}}+\left(\frac{\varphi}{g_{1}}\right)^{\prime}\right) \gamma
\end{aligned}
$$


By (51) and (52), we note that $u^{\prime}=0$ on $U_{0}$. Thus, the above equation provides us with the following equations

$$
\left\{\begin{aligned}
&\left(\frac{1}{g_{1}}\right)^{\prime}=-\frac{\varphi^{\prime}}{\varphi g_{1}}, \\
&\left(\frac{\varphi^{\prime}}{\varphi g_{1}}\right)^{\prime}=\frac{1+u^{2}+\varphi^{2}}{g_{1}}+\varphi, \\
&\left(\frac{\varphi}{g_{1}}\right)^{\prime}=-\frac{\varphi^{\prime}}{g_{1}}
\end{aligned}\right.
$$

as the coefficients of the orthogonal vectors. Comparing the first and the third equations of (54), we can obtain

$$
\varphi^{\prime}=0
$$

which yields that

$$
g_{1}^{\prime}=0 \quad \text { and } \quad\left(\frac{1+u^{2}+\varphi^{2}}{g_{1}}+\varphi\right)=0 \quad \text { on } \quad U_{0} .
$$

Therefore, we see that the function $\varphi$ is nonzero constant on $U_{0}$. The functions $u$ and $g_{1}$ are also constant on $U_{0}$, so is the function $g_{2}$ by virtue of (49). Since $g_{1} \varphi=-\left(1+u^{2}+\varphi^{2}\right)$, we have

$$
f=\frac{1+u^{2}+\varphi^{2}}{(\cos t+u \sin t)^{2}}
$$

and

$$
\mathbf{C}=\frac{1}{1+u^{2}+\varphi^{2}}\left(\varphi \alpha+u \varphi \beta+\left(1+u^{2}\right) \gamma\right)
$$

from (50) and (53), respectively.

According to the results so far, we are ready to construct a ruled surface $M$ in $\mathbb{S}^{3}$ with a pointwise 1-type spherical Gauss map of the second kind which is not totally geodesic, i.e., $\Delta G \neq \mathbf{0}$ : As we saw in Lemma 1, if a ruled surface $M$ in $\mathbb{S}^{3}$ has a pointwise 1-type spherical Gauss map $G$ of the second kind, then $\alpha^{\prime} \wedge \beta^{\prime}=\mathbf{0}$ on $M$. Furthermore, we showed that $q_{s}=0$ on $M$ and hence

$$
\beta(s)=u \alpha(s)+\mathbf{N},
$$

where $\mathbf{N}$ is some constant vector satisfying

$$
\langle\alpha, \mathbf{N}\rangle=-u \text { and }\langle\mathbf{N}, \mathbf{N}\rangle=1+u^{2} .
$$

Since the function $\varphi$ is nonzero constant, we can see that the vector field $\alpha^{\prime \prime}$ given by

$$
\alpha^{\prime \prime}=-\alpha-u \beta+\varphi \gamma
$$

has the constant length $\sqrt{1+u^{2}+\varphi^{2}}$. Thus, we can naturally define a ruled surface $M$ in $\mathbb{S}^{3}\left(\subset \mathbb{E}^{4}\right)$ parameterized by

$$
\begin{aligned}
M: x(s, t) & =\cos t \alpha(s)+\sin t \beta(s) \\
& =(\cos t+u \sin t) \alpha(s)+\sin t \mathbf{N}
\end{aligned}
$$

which has pointwise 1-type spherical Gauss map $G$ of the second kind, that is,

$$
\begin{aligned}
\Delta G & =\frac{1}{(\cos t+u \sin t)^{2}} \gamma^{\prime \prime} \\
& =\frac{1}{(\cos t+u \sin t)^{2}}\left(\varphi\left(1+u^{2}\right) \alpha+u \varphi \mathbf{N}-\varphi^{2} \gamma\right) \\
& =f(G+\mathbf{C}),
\end{aligned}
$$


where we have put

$$
f=\frac{1+u^{2}+\varphi^{2}}{(\cos t+u \sin t)^{2}}
$$

and

$$
\mathbf{C}=\frac{1}{1+u^{2}+\varphi^{2}}\left(\varphi\left(1+u^{2}\right) \alpha+u \varphi \mathbf{N}+\left(1+u^{2}\right) \gamma\right),
$$

respectively.

Meanwhile, we note that the function $\varphi$ is constant on $U_{0}$. By continuity, we see that either $\Delta G=\mathbf{0}$ on $U_{0}$, or it does not. This means that either $G=-\mathrm{C}$ on $U_{0}$ or $x\left(U_{0}\right)$ is an open part of a ruled surface parameterized by (55).

Now, we consider $W=\left\{(s, t) \in U \mid f_{t}(s, t)=0\right\}$, the complement of $U_{0}$, and let $W_{0}=\operatorname{int}(W)$. Then, we will show that if $W_{0}$ is non-empty, the constant vector $\mathbf{C}=\mathbf{0}$ on $W_{0}$, which implies that $W_{0}$ must be empty. Therefore, we have

Lemma 2. Let $M$ be a ruled surface parameterized by (2) in the unit sphere $\mathbb{S}^{3}$. If the spherical Gauss map $G$ of $M$ is of pointwise 1-type of the second kind, i.e., $\Delta G=f(G+C)$ for some non-zero function $f$ and $a$ non-zero constant vector $C$, then we may assume that the function $f_{t}$, the partial derivative of $f$ with respect to $t$, is non-vanishing on $U=\{(s, t) \in I \times J \mid f(s, t) \neq 0\}$, that is, $W_{0}=\varnothing$.

Proof. We suppose that $W_{0}$ is non-empty. From (30), we have

$$
\left(q^{-\frac{7}{2}} P\right)_{t}=\left(q^{-\frac{7}{2}} Q\right)_{t}=0
$$

or, equivalently,

$$
\frac{7}{2} q_{t} P=q P_{t} \quad \text { and } \quad \frac{7}{2} q_{t} Q=q Q_{t}
$$

on $W_{0}$. By a straightforward computation, $\frac{7}{2} q_{t} P=q P_{t}$ of (56) implies

$$
\begin{aligned}
\frac{15}{4} b q_{s} q_{t} \sin t= & \frac{3}{2} b q q_{s t} \sin t+\frac{3}{2} b q q_{s} \cos t+\frac{3}{2} \varphi q q_{t} \cos t+\varphi q^{2} \sin t \\
& +\frac{3}{2}\left(u \varphi+2 b^{\prime}\right) q q_{t} \sin t-q^{2}\left(u \varphi+2 b^{\prime}\right) \cos t
\end{aligned}
$$

We note that

$$
\left\{\begin{aligned}
q & =\cos ^{2} t+2 u \cos t \sin t+w \sin ^{2} t \\
q_{s} & =2 u^{\prime} \cos t \sin t+w^{\prime} \sin ^{2} t \\
q_{t} & =2 u \cos ^{2} t+2(w-1) \cos t \sin t-2 u \sin ^{2} t, \\
q_{s t} & =2 u^{\prime} \cos ^{2} t+2 w^{\prime} \cos t \sin t-2 u^{\prime} \sin ^{2} t .
\end{aligned}\right.
$$

Therefore, we can see that Equation (57) is a polynomial in $\cos ^{5-k} t \sin ^{k} t, k=0,1, \ldots, 5$, with functions of $s$ as coefficients. By considering the linear independence of the trigonometric functions, we get

$$
u \varphi=b^{\prime}
$$

as the coefficients of terms containing ' $\cos ^{5} t^{\prime}$. Thus, the function $Q$ of (32) becomes

$$
Q(s, t)=\frac{3}{2} b q q_{s} \cos t-q^{2}\left(u^{\prime} b-u b^{\prime}-u^{2} \varphi-b^{2} \varphi\right) \sin t
$$


and then $\frac{7}{2} q_{t} Q=q Q_{t}$ of (56) provides

$$
\begin{aligned}
\frac{15}{4} b q_{s} q_{t} \cos t= & \frac{3}{2} b q q_{s t} \cos t-\frac{3}{2} b q q_{s} \sin t+\frac{3}{2}\left(u^{\prime} b-u b^{\prime}-u^{2} \varphi-b^{2} \varphi\right) q q_{t} \sin t \\
& -q^{2}\left(u^{\prime} b-u b^{\prime}-u^{2} \varphi-b^{2} \varphi\right) \cos t .
\end{aligned}
$$

Similarly, using (58), we obtain

$$
2 u^{\prime} b+2 u^{2} \varphi+b^{2} \varphi=0
$$

and

$$
u\left(u^{\prime} b-u^{2} \varphi\right)=0
$$

as the coefficients of the terms containing ' $\cos ^{5} t$ ' and ' $\sin ^{5} t$ ' of (60), respectively. If $u \neq 0$ on some open subset $W_{1}$ of $W_{0}$, then we have

$$
u^{\prime} b=u^{2} \varphi
$$

which helps (61) lead to

$$
\left(4 u^{2}+b^{2}\right) \varphi=0,
$$

or,

$$
\varphi=0 \quad \text { on } \quad W_{1}
$$

because of $u \neq 0$. Since $\varphi=0$, (59) and (62) yield that

$$
b^{\prime}=u^{\prime} b=0 \quad \text { on } W_{1} .
$$

If $b=0$, the function $q=(\cos t+u \sin t)^{2}$ and the spherical Gauss map $G$ is given by

$$
G=A=-\gamma
$$

that is constant because of (8) and (63). In this case, we can easily show that the shape operator on $W_{1}$ is identically zero, which means that $x\left(W_{1}\right)$ is totally geodesic in $\mathbb{S}^{3}$.

Now, we may assume that $b \neq 0$ on $W_{1}$. It follows that $u^{\prime}=0$ of (64) and hence, by continuity, $u$ and $b$ are nonzero constant on $W_{0}$, which tells us that

$$
q_{s}=0 \text { on } W_{0} .
$$

If $u=0$ on $W_{0}$, it is obvious that $b^{\prime}=0$ of (59) and hence $q_{s}=0$ on $W_{0}$. But, in the course of proving $q_{s}=0$, we showed that $\varphi=0$ on $W_{0}$. For the case of $u \neq 0$ on $W_{0}$, we have (63). If $u=0$ on $W_{0}$, (61) yields that $\varphi=0$ on $W_{0}$. Using these results on $W_{0}$, we have

$$
\begin{aligned}
\Delta G= & q^{-\frac{5}{2}}\left\{\left(-\frac{1}{2}\left(q_{t}\right)^{2}+\frac{1}{2} q q_{t t}+q^{2}\right)(\cos t A+\sin t B)\right. \\
& \left.-q\left(\cos t A^{\prime \prime}+\sin t B^{\prime \prime}\right)+\frac{1}{2} q q_{t}(-\sin t A+\cos t B)\right\}, \\
& \left\{\begin{array} { l } 
{ A = - \gamma , } \\
{ A ^ { \prime \prime } = u b \alpha ^ { \prime } + b ^ { 2 } \gamma }
\end{array} \text { and } \left\{\begin{array}{l}
B=b \alpha^{\prime}-u \gamma, \\
B^{\prime \prime}=-b \alpha^{\prime} .
\end{array}\right.\right.
\end{aligned}
$$

By a straightforward computation, we can obtain

$$
\Delta G=\frac{2 b^{2}}{q^{2}} G
$$

which means that the spherical Gauss map $G$ defined on $W_{0}$ cannot be of pointwise 1-type of the second kind. 
By Lemma 2, we conclude that $U=U_{0}$. Then, according to the value of the constant function $\varphi$, that is, zero or not, it follows that either $G=-\mathrm{C}$ on $U$ or $x(U)$ is an open part of a ruled surface parameterized by (55). On the other hand, Theorem 1 shows that if the interior of the set $\{p \in M \mid f(p)=0\}$ of $M$ is non-empty, then it is an open part of a totally geodesic surface in $\mathbb{S}^{3}$. In fact, a totally geodesic surface of $\mathbb{S}^{3}$ has a constant spherical Gauss map. And, we can easily show that the function $\varphi$ defined on a totally geodesic surface of $\mathbb{S}^{3}$ is identically zero for all $s$.

Lemma 3. Let $M$ be a ruled surface in $\mathbb{S}^{3}$ parameterized by (2) with pointwise 1-type spherical Gauss map of the second kind. Then, the function $\varphi(s)=\left\langle\alpha^{\prime \prime}(s), \gamma(s)\right\rangle$ defined on $M$ is constant for all $s$.

By continuity of $\varphi$, we can see that if a ruled surface $M$ of $\mathbb{S}^{3}$ has the spherical Gauss map of pointwise 1-type of the second kind, then we may assume that either $M$ is part of the ruled surface parameterized by (55) or $\Delta G=\mathbf{0}$ on $M$, given by $G=-\mathrm{C}$. Therefore, we have

Theorem 4. Let $M$ be a ruled surface in the unit sphere $\mathbb{S}^{3}$ with a pointwise 1-type spherical Gauss map of the second kind. Then, $M$ is an open part of either the ruled surface parameterized by (55) or a totally geodesic surface.

Example 2. Let us consider a unit speed curve $\alpha$ on $\mathbb{S}^{3}$ and a constant vector $N$ in $\mathbb{E}^{4}$ given by

$$
\alpha(s)=\left(\frac{1}{\sqrt{2}} \cos \sqrt{2} s, \frac{1}{\sqrt{2}} \sin \sqrt{2} s, \frac{1}{\sqrt{2}}, 0\right)
$$

and

$$
N=\left(0,0,1,-\frac{1}{\sqrt{2}}\right) .
$$

Then, we get $\langle\alpha, N\rangle=\frac{1}{\sqrt{2}}$ for all s. By the same argument to get (55), we have

$$
\begin{aligned}
& \beta(s)=\left(-\frac{1}{2} \cos \sqrt{2} s,-\frac{1}{2} \sin \sqrt{2} s, \frac{1}{2},-\frac{1}{\sqrt{2}}\right), \\
& \gamma(s)=\left(-\frac{1}{2} \cos \sqrt{2} s,-\frac{1}{2} \sin \sqrt{2} s, \frac{1}{2}, \frac{1}{\sqrt{2}}\right) .
\end{aligned}
$$

Therefore, the ruled surface $M$ on $\mathbb{S}^{3}$ parameterized by

$$
\begin{aligned}
M: x(s, t)= & \cos t \alpha(s)+\sin t \beta(s) \\
= & \left(\frac{1}{\sqrt{2}}\left(\cos t-\frac{1}{\sqrt{2}} \sin t\right) \cos \sqrt{2} s, \frac{1}{\sqrt{2}}\left(\cos t-\frac{1}{\sqrt{2}} \sin t\right) \sin \sqrt{2} s,\right. \\
& \left.\frac{1}{\sqrt{2}} \cos t+\frac{1}{2} \sin t,-\frac{1}{\sqrt{2}} \sin t\right),
\end{aligned}
$$

has the spherical Gauss map $G$ of the form

$$
G=-\gamma=\left(\frac{1}{2} \cos \sqrt{2} s, \frac{1}{2} \sin \sqrt{2} s,-\frac{1}{2},-\frac{1}{\sqrt{2}}\right),
$$

which satisfies

$$
\Delta G=\frac{2}{\left(\cos t-\frac{1}{\sqrt{2}} \sin t\right)^{2}}\left(G+\left(0,0, \frac{1}{2}, \frac{1}{\sqrt{2}}\right)\right) .
$$

Author Contributions: S.M.J. and Y.H.K. set up the problem and computed the details and J.Q. checked and polished the draft. 
Funding: The first author was supported by the National Research Foundation of Korea (NRF) grant funded by the Korea government (MSIT) (2019R1C1C1006370).

Conflicts of Interest: The authors declare no conflict of interest.

\section{References}

1. Takahashi, T. Minimal immersions of Riemannian manifolds. J. Math. Soc. Jpn. 1966, 18, 380-385. [CrossRef]

2. Chen, B.-Y. Finite-Type Submanifolds and Generalizations; Instituto "Guido Castelnuovo": Rome, Italy, 1985.

3. Chen, B.-Y. Surfaces of finite-type in Euclidean 3-space. Bull. Soc. Math. Belg. 1987, 39, 243-254.

4. Chen, B.-Y.; Dillen, F.; Verstraelen, L.; Vrancken, L. Ruled surfaces of finite-type. Bull. Aust. Math. Soc. 1990, 42, 447-453. [CrossRef]

5. Dillen, F. Ruled submanifolds of finite-type. Proc. Am. Math. Soc. 1992, 114, 795-798. [CrossRef]

6. Kim, Y.H.; Yoon, D.W. Classification of ruled surfaces in Minkowski 3-spaces. J. Geom. Phys. 2004, 49, 89-100. [CrossRef]

7. Kim, Y.H.; Yoon, D.W. On non-developable ruled surfaces in Lorentz-Minkowski 3-spaces. Taiwan. J. Math. 2007, 11, 197-214. [CrossRef]

8. Hasanis, T.; Vlachos, T. A classification of ruled surfaces of finite type in $\mathbb{S}^{3}$. J. Geom. 1994, 50, 84-94. [CrossRef]

9. Chen, B.-Y.; Piccinni, P. Submanifolds with finite-type Gauss map. Bull. Aust. Math. Soc. 1987, 35, 161-186. [CrossRef]

10. Baikoussis, C. Ruled submanifolds with finite-type Gauss map. J. Geom. 1994, 49, 42-45. [CrossRef]

11. Baikoussis, C.; Blair, D.E. On the Gauss map of ruled surfaces. Glasg. Math. J. 1992, 34, 355-359 [CrossRef]

12. Baikoussis, C.; Chen, B.-Y.; Verstraelen, L. Ruled surfaces and tubes with finite-type Gauss map. Tokyo J. Math. 1993, 16, 341-348. [CrossRef]

13. Jang, C. Surfaces with 1-tpye Gauss map. Kodai Math. J. 1996, 19, 388-394. [CrossRef]

14. Kim, D.-S.; Kim, Y.H.; Jung, S.M. Ruled submanifolds with harmonic Gauss map. Taiwan. J. Math. 2014, 18, 53-76. [CrossRef]

15. Kim, D.-S.; Kim, Y.H.; Jung, S.M. Some classifications of ruled submanifolds in Minkowski space and their Gauss map. Taiwan. J. Math. 2014, 18, 1021-1040. [CrossRef]

16. Kim, D.-S.; Kim, Y.H.; Yoon, D.W. Extended B-scrolls and their Gauss maps. Indian J. Pure Appl. Math. 2002, 33, 1031-1040.

17. Kim, Y.H.; Yoon, D.W. Ruled surfaces with finite type Gauss map in Minkowski spaces. Soochow J. Math. 2000, 26, 85-96.

18. Chen, B.-Y.; Choi, M.; Kim, Y.H. Surfaces of revolution with pointwise 1-type Gauss map. J. Korean Math. Soc. 2005, 42, 447-455. [CrossRef]

19. Choi, M.; Kim, Y.H. Characterization of the helicoid as ruled surfaces with pointwise 1-type Gauss map. Bull. Korean Math. Soc. 2001, 38, 753-761.

20. Choi, M.; Kim, D.-S.; Kim, Y.H.; Yoon, D.W. Circular cone and its Gauss map. Colloq. Math. $2012,129$. [CrossRef]

21. Choi, M.; Kim, Y.H.; Yoon, D.W. Classification of ruled surfaces with pointwise 1-type Gauss map. Taiwan. J. Math. 2010, 14, 1297-1308. [CrossRef]

22. Jung, S.M.; Kim, D.-S.; Kim, Y.H.; Yoon, D.W. Gauss maps of ruled submanifolds and applications I. J. Korean Math. Soc. 2016, 53, 1309-1330. [CrossRef]

23. Kim, D.-S.; Kim, Y.H.; June, S.M.; Yoon, D.W. Gauss maps of ruled submanifolds and applications $I I$. Taiwan. J. Math. 2016, 20, 227-242. [CrossRef]

24. Kim, Y.H.; Yoon, D.W. On the Gauss map of ruled surfaces in Minkowski space. Rocky Mt. J. Math. 2005, 35, 1555-1581. [CrossRef]

25. Jung, S.M.; Kim, Y.H. Gauss Map and Its Applications on Ruled Submanifolds in Minkowski Space. Symmetry 2018, 10, 218. [CrossRef]

26. Jung, S.M.; Kim, D.-S.; Kim, Y.H. Minimal ruled submanifolds associated with Gauss map. Taiwan. J. Math. 2018, 22, 567-605. [CrossRef]

27. Obata, M. The Gauss map of immersions of Riemannian manifolds in space of constant curvature. J. Differ. Geom. 1968, 2, 217-223. [CrossRef] 
28. Chen, B.-Y.; Lue, H.-S. Spherical submanifolds with finite type spherical Gauss map. J. Korean Math. Soc. 2007, 44, 407-442. [CrossRef]

29. Osserman, R. Minimal surfaces, Gauss maps, total curvature, eigenvalues estimates and stability. In The Chern Symposium 1979; Springer: New York, NY, USA, 1980; pp. $199-227$.

30. Bekts, B.; Canfes, E.O.; Dursun, U. Pseudo-spherical submanifolds with 1-type pseudo-spherical Gauss map. Results Math. 2017, 71, 867-887. [CrossRef]

31. Bekts, B.; Canfes, E.O.; Dursun, U. Classification of surfaces in a pseudo-sphere with 2-type pseudo-spherical Gauss map. Math. Nachrichten 2017, 290. [CrossRef]

32. Bekts, B.; Dursun, U. On spherical submanifolds with finite type spherical Gauss map. Adv. Geom. 2016, 16, 243-251. [CrossRef]

(C) 2019 by the authors. Licensee MDPI, Basel, Switzerland. This article is an open access article distributed under the terms and conditions of the Creative Commons Attribution (CC BY) license (http:/ / creativecommons.org/licenses/by/4.0/). 